\title{
The maximal length of a gap between $r$-graph Turán densities
}

\author{
Oleg Pikhurko* \\ Mathematics Institute and DIMAP \\ University of Warwick \\ Coventry CV4 7AL, UK \\ 0.Pikhurko@warwick.ac.uk
}

Submitted: Apr 7, 2015; Accepted: Oct 5, 2015; Published: Oct 30, 2015

Mathematics Subject Classifications: 05D05

\begin{abstract}
The Turán density $\pi(\mathcal{F})$ of a family $\mathcal{F}$ of $r$-graphs is the limit as $n \rightarrow \infty$ of the maximum edge density of an $\mathcal{F}$-free $r$-graph on $n$ vertices. Erdös [Israel J. Math 2 (1964):183-190] proved that no Turán density can lie in the open interval $\left(0, r ! / r^{r}\right)$. Here we show that any other open subinterval of $[0,1]$ avoiding Turán densities has strictly smaller length. In particular, this implies a conjecture of Grosu [arXiv: 1403.4653, 2014].
\end{abstract}

\section{Introduction}

Let $\mathcal{F}$ be a (possibly infinite) family of $r$-graphs (that is, $r$-uniform set systems). We call elements of $\mathcal{F}$ forbidden. An $r$-graph $G$ is $\mathcal{F}$-free if no member $F \in \mathcal{F}$ is a subgraph of $G$, that is, we cannot obtain $F$ by deleting some vertices and edges from $G$. The Turán function $\operatorname{ex}(n, \mathcal{F})$ is the maximum number of edges that an $\mathcal{F}$-free $r$-graph on $n$ vertices can have. This is one of the central questions of extremal combinatorics that goes back to the fundamental paper of Turán [16]. We refer the reader to the surveys of the Turán function by Füredi [8], Keevash [12], and Sidorenko [15].

As was observed by Katona, Nemetz, and Simonovits [11], the limit

$$
\pi(\mathcal{F}):=\lim _{n \rightarrow \infty} \frac{\operatorname{ex}(n, \mathcal{F})}{\left(\begin{array}{l}
n \\
k
\end{array}\right)}
$$

exists. It is called the Turán density of $\mathcal{F}$. Let $\Pi_{\infty}^{(r)}$ consist of all possible Turán densities of $r$-graph families and let $\Pi_{\text {fin }}^{(r)}$ be the set of all possible Turán densities when finitely

\footnotetext{
*Supported by ERC grant 306493 and EPSRC grant EP/K012045/1.
} 
many $r$-graphs are forbidden. It is convenient to allow empty forbidden families, so that 1 is also a Turán density. Clearly, $\Pi_{\text {fin }}^{(r)} \subseteq \Pi_{\infty}^{(r)}$. A result of Brown and Simonovits [3, Theorem 1] implies that the topological closure $\mathrm{cl}\left(\Pi_{\text {fin }}^{(r)}\right)$ of $\Pi_{\text {fin }}^{(r)}$ contains $\Pi_{\infty}^{(r)}$ while the converse inclusion was established in [14, Proposition 1]; thus

$$
\Pi_{\infty}^{(r)}=\operatorname{cl}\left(\Pi_{\text {fin }}^{(r)}\right), \quad \text { for every integer } r \geqslant 2 .
$$

For $r=2$, the celebrated Erdős-Stone-Simonovits Theorem [5, 6] determines the Turán density for every family $\mathcal{F}$. In particular, we have

$$
\Pi_{\text {fin }}^{(2)}=\Pi_{\infty}^{(2)}=\left\{\frac{m-1}{m}: m=1,2,3, \ldots, \infty\right\} .
$$

Unfortunately, the Turán function for hypergraphs (that is, $r$-graphs with $r \geqslant 3$ ) is much more difficult to analyse and many problems (even rather basic ones) are wide open.

Fix some $r \geqslant 2$. A gap is an open interval $(a, b) \subseteq(0,1)$ that is disjoint from $\Pi_{\infty}^{(r)}$ (which, by (1), is equivalent to being disjoint from $\Pi_{\text {fin }}^{(r)}$ ). Here we consider $g_{r}$, the maximal possible length of a gap. In other words, $g_{r}$ is the maximal $g$ such that there is a real $a$ with $(a, a+g) \subseteq(0,1) \backslash \Pi_{\infty}^{(r)}$. For example, (2) implies that $g_{2}=1 / 2$. Erdös [4] proved that $\left(0, r ! / r^{r}\right)$ is a gap; in particular, $g_{r} \geqslant r ! / r^{r}$. Here we show that this is equality and every other gap has strictly smaller length.

Theorem 1. For every $r \geqslant 3$, we have that $g_{r}=r ! / r^{r}$ and, furthermore, $\left(0, r ! / r^{r}\right)$ is the only gap of length $r ! / r^{r}$ for $r$-graphs.

In particular we obtain the following result that was conjectured by Grosu [9, Conjecture 10].

Corollary 2. The union of $r$-graph Turán densities over all $r \geqslant 2$ is dense in $[0,1]$, that is, $\operatorname{cl}\left(\cup_{r=2}^{\infty} \Pi_{\infty}^{(r)}\right)=[0,1]$.

The question whether the set $\Pi_{\infty}^{(r)}$ is a well-ordered subset of $([0,1], \leqslant)$ for $r \geqslant 3$ was a famous $\$ 1000$ problem of Erdős that was answered in the negative by Frankl and Rödl [7]. Despite a number of results that followed [7], very little is known about other gaps in $\Pi_{\infty}^{(r)}$ for $r \geqslant 3$. For example, let $g_{r}^{\prime}$ be the second largest gap length, that is, the maximum $g \geqslant 0$ such that $(a, a+g) \subseteq\left(r ! / r^{r}, 1\right) \backslash \Pi_{\infty}^{(r)}$ for some $a$. The computergenerated proof of Baber and Talbot [2] implies that $g_{3}^{\prime} \geqslant 0.0017$. Klas Markström and Fei Song [13] conjectured that $(2 / 7,8 / 27)$ is the (unique) second largest gap for 3 -graphs (and, in particular, $g_{3}^{\prime}=2 / 189$ ). However, not for a single $r \geqslant 4$ is it known, for example, whether $g_{r}^{\prime}$ is zero (i.e. whether $\Pi_{\infty}^{(r)}$ is dense in $\left[r ! / r^{r}, 1\right]$ ).

This paper is organised as follows. In Section 2 we give some definitions and auxiliary results. Theorem 1 is proved in Section 3. We give another proof of Corollary 2 in Section 4. Although the latter proof is not strong enough to prove Theorem 1, its advantage is that it produces explicit elements of $\Pi_{\text {fin }}^{(r)}$ (as opposed to the implicit values of certain maximisation problems returned by the proof in Section 3). So we include both proofs here, even though the second one is longer. 


\section{Preliminaries}

For $n \in \mathbb{N}$, define $[n]:=\{1, \ldots, n\}$. For reals $a \leqslant b$, let $(a, b)$ and $[a, b]$ be respectively the open and closed interval of reals with endpoints $a$ and $b$. The standard $(m-1)$-dimensional simplex is

$$
\mathbb{S}_{m}:=\left\{\boldsymbol{x} \in \mathbb{R}^{m}: x_{1}+\cdots+x_{m}=1, \forall i \in[m] x_{i} \geqslant 0\right\} .
$$

An $r$-pattern is a collection $P$ of $r$-multisets on $[m]$, for some $m \in \mathbb{N}$. (By an $r$-multiset we mean an unordered collection of $r$ elements with repetitions allowed.) Let $V_{1}, \ldots, V_{m}$ be disjoint sets and let $V=V_{1} \cup \cdots \cup V_{m}$. The profile of an $r$-set $X \subseteq V$ (with respect to $\left.V_{1}, \ldots, V_{m}\right)$ is the $r$-multiset on $[m]$ that contains $i \in[m]$ with multiplicity $\left|X \cap V_{i}\right|$. For an $r$-multiset $Y$ on $[m]$, let $Y\left(\left(V_{1}, \ldots, V_{m}\right)\right)$ consist of all $r$-subsets of $V$ whose profile is $Y$. We call this $r$-graph the blow-up of $Y$ (with respect to $V_{1}, \ldots, V_{m}$ ) and the $r$-graph

$$
P\left(\left(V_{1}, \ldots, V_{m}\right)\right):=\bigcup_{Y \in P} Y\left(\left(V_{1}, \ldots, V_{m}\right)\right)
$$

is called the blow-up of $P$. Let the Lagrange polynomial of $P$ be

$$
\lambda_{P}\left(x_{1}, \ldots, x_{m}\right):=r ! \sum_{D \in P} \prod_{i=1}^{m} \frac{x_{i}^{D(i)}}{D(i) !} \in \mathbb{R}\left[x_{1}, \ldots, x_{m}\right],
$$

where $D(i)$ denotes the multiplicity of $i$ in $D$. This definition is motivated by the fact that, for every partition $[n]=V_{1} \cup \cdots \cup V_{m}$, we have that

$$
\left|P\left(\left(V_{1}, \ldots, V_{m}\right)\right)\right|=\lambda_{P}\left(\frac{\left|V_{1}\right|}{n}, \ldots, \frac{\left|V_{m}\right|}{n}\right) \times\left(\begin{array}{l}
n \\
r
\end{array}\right)+O\left(n^{r-1}\right), \quad \text { as } n \rightarrow \infty .
$$

For example, if $r=3, m=3$, and $P$ consists of multisets $\{1,1,2\}$ and $\{1,2,3\}$, then $P\left(\left(V_{1}, \ldots, V_{m}\right)\right)$ contains all triples that have two vertices in $V_{1}$ and one vertex in $V_{2}$ plus all triples with exactly one vertex in each part; here $\lambda_{P}\left(x_{1}, x_{2}, x_{3}\right)=3 x_{1}^{2} x_{2}+6 x_{1} x_{2} x_{3}$.

Let the Lagrangian of $P$ be $\Lambda_{P}:=\max \left\{\lambda_{P}(\boldsymbol{x}): \boldsymbol{x} \in \mathbb{S}_{m}\right\}$, the maximum value of the polynomial $\lambda_{P}$ on the compact set $\mathbb{S}_{m}$. One obvious connection of this parameter to $r$-graph Turán densities is that, if each blow-up of $P$ is $\mathcal{F}$-free, then $\pi(\mathcal{F}) \geqslant \Lambda_{P}$. Also, it is not hard to show that $\Lambda_{P}=\pi(\mathcal{F})$, where $\mathcal{F}$ consists of all $r$-graphs $F$ such that every blow-up of $P$ is $F$-free; thus $\Lambda_{P} \in \Pi_{\infty}^{(r)}$. As shown in [14, Theorem 3], we have in fact that

$$
\Lambda_{P} \in \Pi_{\text {fin }}^{(r)}, \quad \text { for every } r \text {-pattern } P \text {. }
$$

We will use the special case of Muirhead's inequality (see e.g. [10, Theorem 45]) which states that, for any $0 \leqslant i<j \leqslant k$, we have

$$
x^{k+i} y^{k-i}+x^{k-i} y^{k+i} \leqslant x^{k+j} y^{k-j}+x^{k-j} y^{k+j}, \quad \text { for } x, y \geqslant 0 .
$$




\section{Proof of Theorem 1}

Let $r \geqslant 3$. Fix a sufficiently large integer $m=m(r)$ so that $r !\left(\begin{array}{c}m \\ r\end{array}\right) / m^{r}>1-r ! / r^{r}$. Consider $r$-graphs $G_{0}, \ldots, G_{\left(\begin{array}{c}m \\ r\end{array}\right)}$ on $[m]$ such that $G_{0}$ has no edges and, for $i=1, \ldots,\left(\begin{array}{c}m \\ r\end{array}\right)$, the $r$-graph $G_{i}$ is obtained from $G_{i-1}$ by adding a new edge. In other words, we enumerate all $r$-subsets of $[m]$ as $R_{1}, \ldots, R_{\left(\begin{array}{c}m \\ r\end{array}\right)}$ and let $G_{i}:=\left\{R_{1}, \ldots, R_{i}\right\}$. Let

$$
\lambda_{i}(\boldsymbol{x}):=\lambda_{G_{i}}(\boldsymbol{x})=r ! \sum_{D \in G_{i}} \prod_{j \in D} x_{j},
$$

be the Lagrange polynomial of $G_{i}$ and $\Lambda_{i}:=\Lambda_{G_{i}}$ be its Lagrangian, where we view $G_{i}$ as an $r$-pattern. Since $G_{i-1} \subseteq G_{i}$, we have that $\Lambda_{i-1} \leqslant \Lambda_{i}$.

We claim that for every $i \in\left[\left(\begin{array}{c}m \\ r\end{array}\right)\right]$

$$
\Lambda_{i}-\Lambda_{i-1} \leqslant r ! / r^{r}
$$

Indeed, pick $\boldsymbol{x} \in \mathbb{S}_{m}$ with $\Lambda_{i}=\lambda_{i}(\boldsymbol{x})$. Let $R_{i}=\left\{u_{1}, \ldots, u_{r}\right\}$. When we remove the term $r ! x_{u_{1}} \ldots x_{u_{r}}$ from $\lambda_{i}(\boldsymbol{x})$, we get the evaluation of $\lambda_{i-1}$ on $\boldsymbol{x} \in \mathbb{S}_{m}$. By definition, $\Lambda_{i-1} \geqslant \lambda_{i-1}(\boldsymbol{x})$. Also, since $x_{u_{1}}+\cdots+x_{u_{r}} \leqslant 1$, we have $x_{u_{1}} \ldots x_{u_{r}} \leqslant r^{-r}$ by the Geometric-Arithmetic Mean Inequality. Thus we obtain the stated bound:

$$
\Lambda_{i}=\lambda_{i}(\boldsymbol{x})=\lambda_{i-1}(\boldsymbol{x})+r ! x_{u_{1}} \ldots x_{u_{r}} \leqslant \Lambda_{i-1}+r ! / r^{r} .
$$

Also, we have $\Lambda_{\left(\begin{array}{c}m \\ r\end{array}\right)} \geqslant \lambda_{\left(\begin{array}{c}m \\ r\end{array}\right)}\left(\frac{1}{m}, \ldots, \frac{1}{m}\right)=r !\left(\begin{array}{c}m \\ r\end{array}\right) / m^{r}>1-r ! / r^{r}$. This and (3) imply that $g_{r} \leqslant r ! / r^{r}$ (while the above-mentioned result of Erdős [4] gives the converse inequality). Also, if we have equality in (5), then necessarily $x_{u_{1}}=\cdots=x_{u_{r}}=1 / r$, each other $x_{j}$ is zero, and $\Lambda_{i-1}=\lambda_{i-1}(\boldsymbol{x})=0$, implying the uniqueness part of Theorem 1 .

\section{Alternative proof of Corollary 2}

For integers $r, s \geqslant 2$, let $\mathcal{P}_{r, s}$ consist of ordered $s$-tuples $\left(r_{1}, \ldots, r_{s}\right)$ of non-negative integers such that $r_{1} \geqslant \ldots \geqslant r_{s}$ and $r_{1}+\cdots+r_{s}=r$. This set admits a partial order, where $\boldsymbol{x} \succcurlyeq \boldsymbol{y}$ if $\sum_{i=1}^{k} x_{i} \geqslant \sum_{i=1}^{k} y_{i}$ for every $k \in[s-1]$. For example, the (unique) maximal element is $(r, 0, \ldots, 0)$ and the (unique) minimal element is $(\lceil r / s\rceil, \ldots,\lfloor r / s\rfloor)$.

Let $A \subseteq \mathcal{P}_{r, s}$. The set $A$ is called down-closed if $\boldsymbol{y} \in A$ whenever $\boldsymbol{x} \in A$ and $\boldsymbol{x} \succcurlyeq \boldsymbol{y}$. Let $G_{A}$ consist of all $r$-multisets $X$ on $[s]$ such that the multiplicities of $X$ satisfy $\langle X(1), \ldots, X(s)\rangle \in A$, where $\langle\boldsymbol{x}\rangle$ denotes the non-increasing ordering of a vector $\boldsymbol{x}$. Also, we use the shorthand $\lambda_{A}:=\lambda_{G_{A}}$ and $\Lambda_{A}:=\Lambda_{G_{A}}$.

Lemma 3. Let $r, s \geqslant 2$. If $A \subseteq \mathcal{P}_{r, s}$ is down-closed, then $\Lambda_{A}=\lambda_{A}\left(\frac{1}{s}, \ldots, \frac{1}{s}\right)$.

Proof. We use induction on $s$.

First, we prove the base case $s=2$. Let $k:=r / 2$. For $h \geqslant 0$, let $I_{h}$ consist of all integer translates of $k$ whose absolute value is at most $h$, that is, $I_{h}:=(\mathbb{Z}+k) \cap[-h, h]$. 
Also, let $I_{h}^{+}:=I_{h} \cap[0, h]$. (These definitions will allow us to deal with the cases of even and odd $r$ uniformly.) For example, $\mathcal{P}_{r, 2}=\left\{(k+i, k-i): i \in I_{k}^{+}\right\}$.

Take a down-closed set $A \subseteq \mathcal{P}_{r, 2}$. It consists of pairs $(k+i, k-i)$ with $i \in I_{h}^{+}$for some $h \leqslant k$. Then $G_{A}$ consists of all $2 k$-multisets on $\{1,2\}$ that contain 1 with multiplicity $k+i$ for $i \in I_{h}$. By the homogeneity of the polynomials involved, the required inequality can be rewritten as

$$
\sum_{i \in I_{h}}\left(\begin{array}{c}
2 k \\
k+i
\end{array}\right)\left(\frac{x+y}{2}\right)^{2 k}-\sum_{i \in I_{h}}\left(\begin{array}{c}
2 k \\
k+i
\end{array}\right) x^{k+i} y^{k-i} \geqslant 0, \quad \text { for } x, y \geqslant 0 .
$$

We will apply the so-called bunching method where we try to write the desired inequality as a positive linear combination of Muirhead's inequalities (4). If $j \in I_{h}$, then the coefficient in front of $x^{k+j} y^{k-j}$ in (6) is

$$
2^{-2 k}\left(\begin{array}{c}
2 k \\
k+j
\end{array}\right) \sum_{i \in I_{h}}\left(\begin{array}{c}
2 k \\
k+i
\end{array}\right)-\left(\begin{array}{c}
2 k \\
k+j
\end{array}\right) \leqslant 0 .
$$

If $j \in I_{k} \backslash I_{h}$, then the coefficient is $2^{-2 k}\left(\begin{array}{c}2 k \\ k+j\end{array}\right) \sum_{i \in I_{h}}\left(\begin{array}{c}2 k \\ k+i\end{array}\right) \geqslant 0$. Thus, if we group the left-hand side of (6) into terms $x^{k+j} y^{k-j}+x^{k-j} y^{k+j}$, then we get non-positive coefficients for $0 \leqslant j \leqslant h$ followed by non-negative coefficients for $j>h$. Also, the total sum of coefficients is zero because (6) becomes equality for $x=y=1$. Thus we can "bunch" $I_{h}$-terms with $\left(I_{k} \backslash I_{h}\right)$-terms and use (4) to derive the desired inequality (6). This proves the case $s=2$.

Now, let $s \geqslant 3$ and suppose that we have proved the lemma for $s-1$ (and all $r$ ). The function $\lambda_{A}$ is a continuous function on the compact set $\mathbb{S}_{s}$. Let it attain its maximum on some $\boldsymbol{x} \in \mathbb{S}_{s}$. If there is more than one choice, then choose $\boldsymbol{x}$ so that $\Delta:=\sum_{i \neq j}\left|x_{i}-x_{j}\right|$ is minimised. Suppose that $\Delta \neq 0$, say $x_{1} \neq x_{2}$. Note that $\lambda_{A}$ is a homogeneous polynomial of degree $r$, and the coefficient at $x_{1}^{r_{1}} \ldots x_{s}^{r_{s}}$ is $\left(\begin{array}{c}r \\ r_{1}, \ldots, r_{s}\end{array}\right)$ if the ordering $\langle\boldsymbol{r}\rangle$ of $\boldsymbol{r}$ is in $A$ and 0 otherwise.

Fix $j \in\{0, \ldots, r\}$. If we collect all terms in front of $x_{s}^{j}$, we get

$$
\sum_{\substack{\langle\boldsymbol{r}, j\rangle \in A \\
r_{1}+\cdots+r_{s-1}=r-j}}\left(\begin{array}{c}
r \\
r_{1}, \ldots, r_{s-1}, j
\end{array}\right) \prod_{i=1}^{s-1} x_{i}^{r_{i}}=\left(\begin{array}{l}
r \\
j
\end{array}\right) \lambda_{A \backslash j}\left(x_{1}, \ldots, x_{s-1}\right),
$$

where $\langle\boldsymbol{y}, j\rangle$ is obtained from $\boldsymbol{y}$ by appending $j$ and ordering the obtained sequence, while $A \backslash j$ consists of those $\boldsymbol{y} \in \mathcal{P}_{r-j, s-1}$ such that $\langle\boldsymbol{y}, j\rangle \in A$.

Let us show that $A \backslash j \subseteq \mathcal{P}_{r-j, s-1}$ is down-closed. Take arbitrary $\boldsymbol{z} \in A \backslash j$ and $\boldsymbol{y} \preccurlyeq \boldsymbol{z}$. We have to show that $\boldsymbol{y} \in A \backslash j$. Since $A \ni\langle\boldsymbol{z}, j\rangle$ is down-closed, it is enough to show that $\langle\boldsymbol{z}, j\rangle \succcurlyeq\langle\boldsymbol{y}, j\rangle$. We have to compare the sums of the first $i$ terms of $\langle\boldsymbol{z}, j\rangle$ and of $\langle\boldsymbol{y}, j\rangle$ for each $i \in[s-1]$. A problem could arise only if the new entry $j$ was included into these terms for $\langle\boldsymbol{y}, j\rangle$, say as the term number $h \leqslant i$, but not for $\langle\boldsymbol{z}, j\rangle$. Since $\boldsymbol{z} \succcurlyeq \boldsymbol{y}$, we have that $\sum_{f=1}^{h-1} z_{f} \geqslant \sum_{f=1}^{h-1} y_{f}$ (and these are also the initial sums for $\langle\boldsymbol{z}, j\rangle$ and $\langle\boldsymbol{y}, j\rangle$ ). 
Furthermore, each of the subsequent $i-(h-1)$ entries is at least $j$ for $\langle\boldsymbol{z}, j\rangle$ and at most $j$ for $\langle\boldsymbol{y}, j\rangle$. It follows that $\langle\boldsymbol{z}, j\rangle \succcurlyeq\langle\boldsymbol{y}, j\rangle$. Thus $A \backslash j$ is down-closed, as claimed.

By the induction assumption (and since $\lambda_{A \backslash j}$ is a homogeneous polynomial), we have that $\lambda_{A \backslash j}\left(x_{1}, \ldots, x_{s-1}\right) \leqslant \lambda_{A \backslash j}\left(\frac{1-x_{s}}{s-1}, \ldots, \frac{1-x_{s}}{s-1}\right)$. Thus

$$
\Lambda_{A}=\lambda_{A}(\boldsymbol{x})=\sum_{j=0}^{r}\left(\begin{array}{l}
r \\
j
\end{array}\right) \lambda_{A \backslash j}\left(x_{1}, \ldots, x_{s-1}\right) x_{s}^{j} \leqslant \lambda_{A}\left(\frac{1-x_{s}}{s-1}, \ldots, \frac{1-x_{s}}{s-1}, x_{s}\right) .
$$

Clearly, the sum $\sum_{i=1}^{s-1}\left|x_{s}-x_{i}\right|$ does not increase if we replace each of $x_{1}, \ldots, x_{s-1}$ by their arithmetic mean $\left(1-x_{s}\right) /(s-1)$. Since $x_{1} \neq x_{2}$, we have found another optimal element of $\mathbb{S}_{s}$ with strictly smaller $\Delta$, a contradiction. The lemma is proved.

Fix some enumeration $\mathcal{P}_{r, r}=\left\{R_{1}, \ldots, R_{t}\right\}$ such that if $R_{i} \succcurlyeq R_{j}$ then $i \geqslant j$. For $j \in\{0, \ldots, t\}$, let $A_{j}:=\left\{R_{i}: i \in[j]\right\}$. Thus, for example, $A_{0}=\varnothing$ and $A_{t}=\mathcal{P}_{r, r}$. By $(3)$, $\Pi_{\text {fin }}^{(r)}$ contains all of the following numbers:

$$
0=\Lambda_{A_{0}} \leqslant \Lambda_{A_{1}} \leqslant \cdots \leqslant \Lambda_{A_{t}}=1 .
$$

Let us show that $\max \left\{\Lambda_{A_{i}}-\Lambda_{A_{i-1}}: i \in[t]\right\}=o(1)$ as $r \rightarrow \infty$. By definition, each $A_{j} \subseteq \mathcal{P}_{r, r}$ is down-closed. Thus, by Lemma 3 the difference $\Lambda_{A_{i}}-\Lambda_{A_{i-1}}$ is the probability that, when $r$ balls are uniformly and independently distributed into $r$ cells, the ordered ball distribution is given by $R_{i}$. Expose the first $r-m$ balls, where, for example, $m:=\lfloor\log r\rfloor$. Let $k$ be the number of empty cells. Its expected value is $r(1-1 / r)^{r-m}=\left(\mathrm{e}^{-1}+o(1)\right) r$. By Azuma's inequality (see e.g. [1, Theorem 7.2.1]), we have whp (i.e. with probability $1-o(1)$ as $r \rightarrow \infty)$ that $k$ is in $I:=[r / 4,3 r / 4]$. Assume that $k \in I$ and expose the remaining $m$ balls. Let $J$ be the number of balls that land inside the $k$ cells that were empty after the first round. The probability that $J=j$ for any particular integer $j \in[m / 8,7 m / 8]$ is

$$
\begin{aligned}
\left(\begin{array}{c}
m \\
j
\end{array}\right)\left(\frac{k}{r}\right)^{j}\left(\frac{r-k}{r}\right)^{m-j} & =(1+o(1)) \sqrt{\frac{m}{2 \pi j(m-j)}}\left(\frac{m k}{j r}\right)^{j}\left(\frac{m(r-k)}{(m-j) r}\right)^{m-j} \\
& \leqslant(1+o(1)) \sqrt{\frac{m}{2 \pi j(m-j)}}=o(1),
\end{aligned}
$$

where we used Stirling's formula and the Arithmetic-Geometric Mean Inequality. On the other hand, we have whp that $m / 8 \leqslant J \leqslant 7 m / 8$ (by Azuma's inequality and our assumption $k \in I$ ) and that the last $m$ balls all go into different cells (since $m^{2}=o(r)$ ). Once the first $r-m$ balls are exposed and we condition on the event that the last $m$ balls all land into distinct cells, there is at most one value of $J$ for which the final ball distribution is $R_{i}$. Thus the probability of getting $R_{i}$ is $o(1)$ uniformly in $i$, as desired. This finishes the second proof of Corollary 2.

\section{Acknowledgements}

The author would like to thank Codrut Grosu for helpful comments. 


\section{References}

[1] N. Alon and J. Spencer, The probabilistic method, 3d ed., Wiley Interscience, 2008.

[2] R. Baber and J. Talbot, Hypergraphs do jump, Combin. Probab. Computing 20 (2011), 161-171.

[3] W. G. Brown and M. Simonovits, Digraph extremal problems, hypergraph extremal problems and the densities of graph structures, Discrete Math. 48 (1984), 147-162.

[4] P. Erdős, On extremal problems of graphs and generalized graphs, Israel J. Math. 2 (1964), 183-190.

[5] P. Erdős and M. Simonovits, A limit theorem in graph theory, Stud. Sci. Math. Hungar. (1966), 51-57.

[6] P. Erdős and A. H. Stone, On the structure of linear graphs, Bull. Amer. Math. Soc. 52 (1946), 1087-1091.

[7] P. Frankl and V. Rödl, Hypergraphs do not jump, Combinatorica 4 (1984), 149-159.

[8] Z. Füredi, Turán type problems, Surveys in Combinatorics (A. D. Keedwell, ed.), London Math. Soc. Lecture Notes Ser., vol. 166, Cambridge Univ. Press, 1991, pp. 253300 .

[9] C. Grosu, On the algebraic and topological structure of the set of Turán densities, arXiv: 1403.4653, 2014.

[10] G. H. Hardy, J. E. Littlewood, and G. Pólya, Inequalities, Cambridge Univ. Press, 1952, 2d ed.

[11] G. O. H. Katona, T. Nemetz, and M. Simonovits, On a graph problem of Turán (In Hungarian), Mat. Fiz. Lapok 15 (1964), 228-238.

[12] P. Keevash, Hypergraph Turán problem, Surveys in Combinatorics (R. Chapman, ed.), London Math. Soc. Lecture Notes Ser., vol. 392, Cambridge Univ. Press, 2011, pp. 83-140.

[13] K. Markström, Turán densities for 3-graphs, Talk at the 25th British Combinatorial Conference, 6-10 July 2015.

[14] O. Pikhurko, On possible Turán densities, Israel J. Math. 201 (2014), 415-454.

[15] A. Sidorenko, What we know and what we do not know about Turán numbers, Graphs Combin. 11 (1995), 179-199.

[16] P. Turán, On an extremal problem in graph theory (in Hungarian), Mat. Fiz. Lapok 48 (1941), 436-452. 